\title{
On the ground electronic states of copper silicide and its ions
}

\author{
Alexander I. Boldyrev and Jack Simons \\ Department of Chemistry, The University of Utah, Salt Lake City, Utah 84112 \\ J. J. Scherer, J. B. Paul, C. P. Collier, and R. J. Saykally \\ Department of Chemistry, University of California, Berkeley, Berkeley, California 94720
}

(Received 3 April 1997; accepted 7 January 1998)

\begin{abstract}
The low-lying electronic states of $\mathrm{SiCu}, \mathrm{SiCu}^{+}$, and $\mathrm{SiCu}^{-}$have been studied using a variety of high-level ab initio techniques. As expected on the basis of simple orbital occupancy and bond forming for $\mathrm{Si}\left(s^{2} p^{2}\right)+\mathrm{Cu}\left(s^{1}\right)$ species, ${ }^{2} \Pi_{r},{ }^{1} \Sigma^{+}$, and ${ }^{3} \Sigma^{-}$states were found to be the ground electronic states for $\mathrm{SiCu}, \mathrm{SiCu}^{+}$, and $\mathrm{SiCu}^{-}$, respectively; the ${ }^{2} \Pi_{r}$ state is not that suggested in most recent experimental studies. All of these molecules were found to be quite strongly bound although the bond lengths, bond energies, and harmonic frequencies vary slightly among them, as a result of the nonbonding character of the $2 \pi$-MO (molecular orbital) [composed almost entirely of the Si $3 p$-AO (atomic orbital)], the occupation of which varies from 0 to 2 within the ${ }^{1} \Sigma^{+},{ }^{2} \Pi_{r}$, and ${ }^{3} \Sigma^{-}$series. The neutral $\mathrm{SiCu}$ is found to have bound excited electronic states of ${ }^{4} \Sigma^{-},{ }^{2} \Delta,{ }^{2} \Sigma^{+}$, and ${ }^{2} \Pi_{i}$ symmetry lying $0.5,1.2,1.8$, and $3.2 \mathrm{eV}$ above the ${ }^{2} \Pi_{r}$ ground state. It is possible but not yet certain that the ${ }^{2} \Pi_{i}$ state is, in fact, the " $B$ state" observed in the recent experimental studies by Scherer, Paul, Collier, and Saykally. () 1998 American Institute of Physics.
\end{abstract}

[S0021-9606(98)00914-3]

\section{INTRODUCTION}

In recent cavity ringdown laser absorption spectroscopy (CRLAS) studies of Scherer et al. ${ }^{1}$ the first copper silicide spectra were measured in the UV-vis region from 410 to 380 $\mathrm{nm}$. The observed UV (ultraviolet) band system (labeled as $B-X)$ consisted of a single vibronic progression in the upper electronic state. The $B$ label for the upper state was chosen based on the likelihood that the band origin was too high in energy to correspond to the first excited state, combined with the fact that additional unassigned spectra were measured to the red only under conditions which favored $\mathrm{CuSi}$ production. In that initial work, rotational resolution was achieved, yet no $Q$-branches were observed. A ${ }^{2} \Sigma$ state assignment was thus given for both the upper and lower states observed from several factors, although the likelihood of a ${ }^{2} \Pi$ ground state is strongly indicated based on simple orbital occupancy considerations. A primary focus of the present work has been to sort out this confusion surrounding the ${ }^{2} \Pi$ and ${ }^{2} \Sigma$ state orderings.

Specifically, a basic description of the ground-state bonding in $\mathrm{CuSi}$ involves combining ground-state $\mathrm{Cu}$ atom $\left(3 d^{10} 4 s^{1}\right.$ ) with the $3 s^{2} 3 p^{2}$ configuration of $\mathrm{Si}$. In the $L-S$ coupling limit, this amounts to finding the states resulting from combining a ${ }^{2} S \mathrm{Cu}$ atom with the ${ }^{3} P,{ }^{1} D$, and ${ }^{1} S$ states of the Si atom. States resulting from the combination of the lowest ${ }^{2} S$ and ${ }^{3} P$ terms are of ${ }^{2} \Sigma^{-},{ }^{2} \Pi,{ }^{4} \Sigma^{-}$, and ${ }^{4} \Pi$ symmetry, while states resulting from the ${ }^{2} S$ and ${ }^{1} D$ combination would possess ${ }^{2} \Sigma^{+},{ }^{2} \Pi$, and ${ }^{2} \Delta$ symmetry. Finally, the addition of ${ }^{2} S$ and ${ }^{1} S$ asymptotes produces only a ${ }^{2} \Sigma^{+}$state.

Since the ground state of $\mathrm{Si}$ is ${ }^{3} \mathrm{P}$, one would predict the ground state of $\mathrm{SiCu}$ to be of ${ }^{2} \Pi$ symmetry, derived from pairing the $\mathrm{Cu} s$-electron with a $p_{\sigma}$ electron of $\mathrm{Si}$ while retaining one $p_{\pi}$ electron. However, in the event that bond- ing arises from excited $\mathrm{Si}$ asymptotes, $\mathrm{a}^{2} \Sigma^{+}$state would be more likely. The CRLAS data ${ }^{1}$ obtained were consistent with either of these possibilities. However, spectral congestion in the origin region precluded definitive assignment. Due to the lack of observed $Q$-branches, it was only possible to rigorously assign these bands as belonging to $\Delta L(\Omega)=0$ (i.e., $\Sigma \rightarrow \Sigma$ or $\Pi \rightarrow \Pi)$ transitions. In the event of the $L>0 \rightarrow L$ $>0$ assignment, one would expect a very weak $Q$-branch, which would rapidly decrease in transition strength with increasing $J$. Given the $50 \mathrm{~K}$ rotational temperatures typically achieved in the silane-seeded laser ablation beams, $Q$-branches of this type could have easily been obscured by other $P$ and $R$-branch lines from the three most abundant $\mathrm{CuSi}$ isotopes. For these reasons, Scherer et al. ${ }^{1}$ adopted the ${ }^{2} \Sigma$ lower state assignment, again recognizing the possibility that the observed bands could instead be due to $\Pi \rightarrow \Pi$ transitions, wherein the expected weak $Q$-branches might not be resolved. Finally, it is possible that the transitions observed actually originate from an excited $\mathrm{CuSi}$ state, and do not, in fact, originate from the ground state.

\section{COMPUTATIONAL DETAILS}

The bond lengths and harmonic vibrational frequencies of the lowest electronic states of $\mathrm{SiCu}, \mathrm{SiCu}^{+}$, and $\mathrm{SiCu}^{-}$ were optimized using analytical gradients and polarized split-valence basis sets of $6-311++\mathrm{G}(d, f)$ quality $^{2-7}[++$ denotes diffuse $s, p$-functions on $\mathrm{Si}$ and diffuse $d$-functions on $\mathrm{Cu}$ and $(d, f)$ denotes polarization $d$-functions on $\mathrm{Si}$ and polarization $f$-functions on $\mathrm{Cu}$ ] within the density functional theory (B3LYP), ${ }^{8,9}$ second-order M $\not l l e r-P l e s s e t$ perturba- 
TABLE I. Calculated molecular properties of the lowest bound $\mathrm{SiCu}, \mathrm{SiCu}^{+}$, and $\mathrm{SiCu}-\mathrm{States}$.

\begin{tabular}{|c|c|c|}
\hline $\operatorname{SiCu}\left({ }^{2} \Pi_{r}\right)$ & $\operatorname{SiCu}\left({ }^{4} \Sigma^{-}\right)$ & $\mathrm{SiCu}\left({ }^{2} \Pi_{i}\right)$ \\
\hline $1 \sigma^{2} 1 \delta^{4} 1 \pi^{4} 2 \sigma^{2} 3 \sigma^{2} 2 \pi^{1}$ & $1 \sigma^{2} 1 \delta^{4} 1 \pi^{4} 2 \sigma^{2} 3 \sigma^{1} 2 \pi^{2}$ & $1 \sigma^{2} 1 \delta^{4} 1 \pi^{4} 2 \sigma^{2} 3 \sigma^{0} 2 \pi^{3}$ \\
\hline QCISD/6-311++ $\mathrm{G}(d, f)$ & $\mathrm{QCISD} / 6-311++\mathrm{G}(d, f)$ & QCISD/6-311++ $\mathrm{G}(d, f)$ \\
\hline$E_{\mathrm{QCISD}}=-1928.172526$ & $E_{\mathrm{QCISD}}=-1928.156300$ & $E_{\mathrm{QCISD}}=-1928.041555$ \\
\hline$R_{e}(\mathrm{Si}-\mathrm{Cu})=2.242 \AA$ & $R_{e}(\mathrm{Si}-\mathrm{Cu})=2.226 \AA$ & $R_{e}(\mathrm{Si}-\mathrm{Cu})=2.176 \AA$ \\
\hline$\omega_{e}=336 \mathrm{~cm}^{-1}$ & $\omega_{e}=320 \mathrm{~cm}^{-1}$ & \\
\hline$\left\langle S^{2}\right\rangle=0.760$ & $\left\langle S^{2}\right\rangle=3.761$ & $\left\langle S^{2}\right\rangle=0.869$ \\
\hline$T_{e, \mathrm{QCISD}}=0.0 \mathrm{eV}$ & $T_{e, \mathrm{QCISD}}=0.442 \mathrm{eV}$ & $T_{e, \mathrm{QCISD}}=3.564 \mathrm{eV}$ \\
\hline$Q^{\mathrm{NPA}}(\mathrm{Si})=-0.073 \mathrm{e}$ & $Q^{\mathrm{NPA}}(\mathrm{Si})=-0.495 \mathrm{e}$ & $Q^{\mathrm{NPA}}(\mathrm{Si})=-0.738 \mathrm{e}$ \\
\hline$\mu_{e}=1.396 \mathrm{D}$ & $\mu_{e}=3.253 \mathrm{D}$ & $\mu_{e}=3.381 \mathrm{D}$ \\
\hline $\mathrm{QCISD}(\mathrm{T}) / 6-311++\mathrm{G}(2 d, 2 f)$ & $\operatorname{QCISD}(\mathrm{T}) / 6-311++\mathrm{G}(2 d, 2 f)$ & $\operatorname{QCISD}(\mathrm{T}) / 6-311++\mathrm{G}(2 d, 2 f)$ \\
\hline$E_{\mathrm{QCISD}(\mathrm{T})}=-1928.255029^{\mathrm{a}}$ & $E_{\mathrm{QCISD}(\mathrm{T})}=-1928.237914^{\mathrm{a}}$ & $E_{\mathrm{QCISD}(\mathrm{T})}=-1928.136847^{\mathrm{a}}$ \\
\hline$T_{e, \mathrm{QCISD}(\mathrm{T})}=0.0 \mathrm{eV}$ & $T_{e, \mathrm{QCISD}(\mathrm{T})}=0.466 \mathrm{eV}$ & $T_{e, \mathrm{QCISD}(\mathrm{T})}=3.216 \mathrm{eV}$ \\
\hline $\mathrm{SiCu}^{+}\left({ }^{1} \Sigma^{+}\right)$ & $\mathrm{SiCu}^{+}\left({ }^{3} \Pi_{r}\right)$ & $\mathrm{SiCu}^{+}\left({ }^{3} \Sigma^{-}\right)$ \\
\hline $1 \sigma^{2} 1 \delta^{4} 1 \pi^{4} 2 \sigma^{2} 3 \sigma^{2} 2 \pi^{0}$ & $1 \sigma^{2} 1 \delta^{4} 1 \pi^{4} 2 \sigma^{2} 3 \sigma^{1} 2 \pi^{1}$ & $1 \sigma^{2} 1 \delta^{4} 1 \pi^{4} 2 \sigma^{2} 3 \sigma^{0} 2 \pi^{2}$ \\
\hline QCISD/6-311++ G(d,f $)$ & $\mathrm{QCISD} / 6-311++\mathrm{G}(d, f)$ & QCISD/6-311++ $\mathrm{G}(d, f)$ \\
\hline$E_{\mathrm{QCISD}}=-1927.924676$ & $E_{\mathrm{QCISD}}=-1927.904267$ & $E_{\mathrm{QCISD}}=-1927.848560$ \\
\hline$R_{e}(\mathrm{Si}-\mathrm{Cu})=2.257 \AA$ & $R_{e}(\mathrm{Si}-\mathrm{Cu})=2.357 \AA$ & $R_{e}(\mathrm{Si}-\mathrm{Cu})=2.407 \AA$ \\
\hline$\omega_{e}=358 \mathrm{~cm}^{-1}$ & $\begin{array}{l}\omega_{e}=240 \mathrm{~cm}^{-1} \\
\left\langle S^{2}\right\rangle=2.014\end{array}$ & $\begin{array}{l}\omega_{e}=179 \mathrm{~cm}^{-1} \\
\left\langle S^{2}\right\rangle=2.107\end{array}$ \\
\hline$T_{e, \mathrm{QCISD}}=0.0 \mathrm{eV}$ & $T_{e, \mathrm{QCISD}}=0.555 \mathrm{eV}$ & $T_{e, \mathrm{QCISD}}=2.071 \mathrm{eV}$ \\
\hline$Q^{\mathrm{NPA}}(\mathrm{Si})=+0.633 \mathrm{e}$ & $Q^{\mathrm{NPA}}(\mathrm{Si})=+0.297 \mathrm{e}$ & $Q^{\mathrm{NPA}}(\mathrm{Si})=+0.054 \mathrm{e}$ \\
\hline $\mathrm{QCISD}(\mathrm{T}) / 6-311++\mathrm{G}(2 d, 2 f)$ & $\operatorname{QCISD}(\mathrm{T}) / 6-311++\mathrm{G}(2 d, 2 f)$ & $\mathrm{QCISD}(\mathrm{T}) / 6-311++\mathrm{G}(2 d, 2 f)$ \\
\hline$E_{\mathrm{QCISD}(\mathrm{T})}=-1927.997449^{\mathrm{a}}$ & $E_{\mathrm{QCISD}(\mathrm{T})}=-1927.979082^{\mathrm{a}}$ & $E_{\mathrm{QCISD}(\mathrm{T})}=-1927.924404^{\mathrm{a}}$ \\
\hline$T_{e, \mathrm{QCISD}(\mathrm{T})}=0.0 \mathrm{eV}$ & $T_{e, \mathrm{QCISD}(\mathrm{T})}=0.501 \mathrm{eV}$ & $T_{e, \mathrm{QCISD}(\mathrm{T})}=1.988 \mathrm{eV}$ \\
\hline $\mathrm{SiCu}^{-}\left({ }^{3} \Sigma^{-}\right)$ & $\mathrm{SiCu}^{-}\left({ }^{5} \Sigma^{-}\right)$ & $\mathrm{SiCu}^{-}\left({ }^{3} \Pi_{r}\right)$ \\
\hline $1 \sigma^{2} 1 \delta^{4} 1 \pi^{4} 2 \sigma^{2} 3 \sigma^{2} 2 \pi^{2}$ & $1 \sigma^{2} 1 \delta^{4} 1 \pi^{4} 2 \sigma^{2} 3 \sigma^{1} 2 \pi^{2} 4 \sigma^{1}$ & $1 \sigma^{2} 1 \delta^{4} 1 \pi^{4} 2 \sigma^{2} 3 \sigma^{2} 2 \pi^{1} 4 \sigma^{1}$ \\
\hline QCISD/6-311++ $\mathrm{G}(d, f)$ & $\mathrm{QCISD} / 6-311++\mathrm{G}(d, f)$ & $\mathrm{QCISD} / 6-311++\mathrm{G}(d, f)$ \\
\hline$E_{\mathrm{QCISD}}=-1928.217470$ & $E_{\mathrm{QCISD}}=-1928.1826007$ & $E_{\mathrm{QCISD}}=-1928.187167$ \\
\hline$R_{e}(\mathrm{Si}-\mathrm{Cu})=2.262 \AA$ & $R_{e}(\mathrm{Si}-\mathrm{Cu})=2.330 \AA$ & $R_{e}(\mathrm{Si}-\mathrm{Cu})=2.278 \AA$ \\
\hline$\omega_{e}=283 \mathrm{~cm}^{-1}$ & $\omega_{e}=236 \mathrm{~cm}^{-1}$ & \\
\hline$\left\langle S^{2}\right\rangle=2.040$ & $\left\langle S^{2}\right\rangle=6.005$ & $\left\langle S^{2}\right\rangle=2.014$ \\
\hline$T_{e, \mathrm{QCISD}}=0.0 \mathrm{eV}$ & $T_{e, \mathrm{QCISD}}=0.949 \mathrm{eV}$ & $T_{e, \mathrm{QCISD}}=0.825 \mathrm{eV}$ \\
\hline$Q^{\mathrm{NPA}}(\mathrm{Si})=-0.870 \mathrm{e}$ & $Q^{\mathrm{NPA}}(\mathrm{Si})=-0.895 \mathrm{e}$ & $Q^{\mathrm{NPA}}(\mathrm{Si})=-0.715 \mathrm{e}$ \\
\hline $\mathrm{QCISD}(\mathrm{T}) / 6-311++\mathrm{G}(2 d, 2 f)$ & $\operatorname{QCISD}(\mathrm{T}) / 6-311++\mathrm{G}(2 d, 2 f)$ & $\mathrm{QCISD}(\mathrm{T}) / 6-311++\mathrm{G}(2 d, 2 f)$ \\
\hline$E_{\mathrm{QCISD}(\mathrm{T})}=-1928.307359^{\mathrm{a}}$ & $E_{\mathrm{QCISD}(\mathrm{T})}=-1928.267148^{\mathrm{a}}$ & $E_{\mathrm{QCISD}(\mathrm{T})}=-1928.265514^{\mathrm{a}}$ \\
\hline$T_{e, \mathrm{QCISD}(\mathrm{T})}=0.0 \mathrm{eV}$ & $T_{e, \mathrm{QCISD}(\mathrm{T})}=1.094 \mathrm{eV}$ & $T_{e, \mathrm{QCISD}(\mathrm{T})}=1.139 \mathrm{eV}$ \\
\hline
\end{tabular}

${ }^{\mathrm{a}}$ Optimized at QCISD/6-311++G( $\left.d, f\right)$.

tion theory (MP2),${ }^{10}$ and quadratic configuration interaction methods including all single- and double-excitation (QCISD). ${ }^{11}$ The energies of the lowest electronic states were then refined at the higher $\mathrm{QCISD}(\mathrm{T}) / 6-311++\mathrm{G}(2 d, 2 f)$ level using the QCISD/6-311++ $\mathrm{G}(d, f)$ optimized geometries. Only the findings at the QCISD and QCISD(T) levels are reported since they are our best predictions. The unrestricted MP2 (UMP2) wave functions for open-shell systems were spin-projected to produce purer spectroscopic states (PMP2). ${ }^{12}$ All calculations were carried out with the GAUSSIAN $94^{13}$ suite of programs and core orbitals were ( $\mathrm{Si}$ $1 s-2 p$ and $\mathrm{Cu} 1 s-3 p$ ) were frozen in all correlated calculations; the $\mathrm{Cu} d$ electrons were included, not frozen.

Because some states (e.g., the ${ }^{2} \Sigma^{+}$and ${ }^{2} \Delta$ ) cannot be properly described by a single determinant, the geometries and harmonic vibrational frequencies of $\mathrm{SiCu}$ were also studied using the complete active space self-consistent field-multireference configuration interaction method, including all single and double excitations from the reference configurations (CASSCF-MRCISD) $)^{14,15}$ using the ANO basis set of Pierloot et al. ${ }^{16}$ within the MOLCAS-3 program. ${ }^{17}$
The CASSCF/MRCISD energies were corrected by the Davidson method ${ }^{18,19}$ [CASSCF/MRCISD(Q)] to account for the quadruple and higher order excited configurations. Finally, the Vibrot program from the MOLCAS-3 package was used for numerically solving the one-dimensional Schrödinger equation to obtain the molecular vibrational parameters at this CASSCF-MRCISD(Q) level. Due to the computational demands of this approach, the $\mathrm{Cu} 3 d$ electrons could not be included in the correlation energy calculations. As a result, the CASSCF/MRCISD data are not as accurate as the QCISD and QCISD(T) predictions, but are essential for states with more than one dominant determinant.

\section{RESULTS AND DISCUSSIONS}

The results of our calculations for $\mathrm{SiCu}, \mathrm{SiCu}^{+}$, and $\mathrm{SiCu}^{-}$are presented in Table I. The dissociation energies for $\mathrm{SiCu}, \mathrm{SiCu}^{+}$, and $\mathrm{SiCu}^{-}$and the ionization potential and electron affinity for $\mathrm{SiCu}$ are summarized in Table II. 


\section{A. $\mathrm{SiCu}$}

We studied what are probably the three lowest electronic states: ${ }^{2} \Pi_{r}\left(1 \sigma^{2} 1 \delta^{4} \pi^{4} 2 \sigma^{2} 3 \sigma^{2} 2 \pi^{1}\right),{ }^{4} \Sigma^{-}\left(1 \sigma^{2} 1 \delta^{4} 1 \pi^{4} 2 \sigma^{2} 3\right.$ $\left.\sigma^{1} 2 \pi^{2}\right),{ }^{2} \Pi_{i}\left(1 \sigma^{2} 1 \delta^{4} 1 \pi^{4} 2 \sigma^{2} ; 3 \sigma^{0} 2 \pi^{3}\right)$ at the B3LYP, MP2, and QCISD levels of theory. The ${ }^{2} \Delta\left[1 \sigma^{2} 1 \delta^{4} 1 \pi^{4} 2 \sigma^{2} 3 \sigma^{1}(2\right.$ $\left.\left.\pi_{x}^{2}-2 \pi_{y}^{2}\right)\right]$ and ${ }^{2} \Sigma^{+}\left[1 \sigma^{2} 1 \delta^{4} 1 \pi^{4} 2 \sigma^{2} 3 \sigma^{1}\left(2 \pi_{x}^{2}+2 \pi_{y}^{2}\right)\right]$ states were examined at the CASSCF/MRCISD level of theory because of their intrinsically multideterminential nature. Notice that we number only the valence orbitals as a result of which the $\mathrm{Cu}-\mathrm{Si} \sigma$-bond is the $2 \sigma$ orbital. The states considered involve double occupancy of the $3 d$ orbitals of $\mathrm{Cu}$, as well as for the bonding $2 \sigma$-MO with the three other valence electrons distributed throughout the $3 \sigma, 4 \sigma$, and $2 \pi$ valence orbitals.

The ${ }^{2} \Pi_{r}$ state was found to be the ground state at all three (B3LYP, MP2, and QCISD) levels of theory, and all three methods give very similar bond lengths $(2.24 \AA)$, harmonic frequencies $\left(\approx 336 \mathrm{~cm}^{-1}\right)$ and dissociation energies $(\approx 1.8 \mathrm{eV})$. In this state, we find the unpaired spin density to be located completely on the silicon atom, we find it to be very ionic $\left(\mu_{e}=1.4 \mathrm{D}\right)$ and the calculated harmonic frequency and dissociation energy both suggest strong bonding in this ground state of $\mathrm{SiCu}$. At the CASSCF/MRCISD and CASSCF/MRCISD(Q) levels of theory, the calculated bond lengths are longer: 2.44 and $2.52 \AA$ and the frequencies are softer: 249 and $235 \mathrm{~cm}^{-1}$, respectively, than for our most accurate data at the QCISD level of theory. This is a result of neglecting the $\mathrm{Cu} 3 d$ electrons in the CASSCF/MRCISD correlation calculations, not because of any multiconfigurational nature of this state for which the Hartree-Fock (HF) configuration is dominate $\left(C_{\mathrm{HF}}=0.93\right)$.

A ${ }^{4} \Sigma^{-}$state arising from promoting one electron from the nonbonding $3 \sigma$-MO into the $2 \pi$-MO is the lowest excited state with a $0.47 \mathrm{eV}$ excitation energy the QCISD(T)/6-311++G(2d,2f ) level of theory. This state is more ionic $\left(\mu_{e}=3.3 \mathrm{D}\right)$ than the ground state and has most (i.e., $2.4 e$ ) of the unpaired electron density located mostly on the silicon atom. Both the ground and this first excited states are strongly bound with very similar bond lengths and harmonic frequencies.

A bound ${ }^{2} \Pi_{i}$ doublet state arising from promotion of two electrons from the $3 \sigma$-MO into the $2 \pi$-MO is substantially higher $[\sim 3.2 \mathrm{eV}$ at $\mathrm{QCISD}(\mathrm{T}) / 6-311++\mathrm{G}(2 d, 2 f)]$ in energy, is even more ionic ( $\left.\mu_{e}=3.4 \mathrm{D}\right)$ and does lie in the region where Scherer et al. ${ }^{1}$ observed a $\Delta L(\Omega)=0$ transition. It is possible that the ${ }^{2} \Pi_{i}$ state is, in fact, the " $B$ state" observed in that study, since the ground state $\left({ }^{2} \Pi\right)$ has the same symmetry. To more carefully examine this possibility we performed CASSCF/MRCISD calculations for the second and third roots of ${ }^{2} \Pi$ symmetry (the ${ }^{2} \Pi_{r}$ ground state being the first root). The second root was found to be very multiconfigurational, to have the dominant $C\left(2 \sigma^{2} 3 \sigma^{\alpha} 4 \sigma^{\alpha} 2\right.$ $\left.\pi^{\beta}\right)=0.72$ configuration in the MRCISD expansion, and to be repulsive at CASSCF/MRCISD level of theory. The third root is also quite multiconfigurational with the dominant $C\left(2 \sigma^{2} 3 \sigma^{1} 4 \sigma^{0} 2 \pi^{3}\right)=0.63$ configuration in the MRCISD expansion, and has a minimum at $r_{e}=2.41 \AA$ with the harmonic frequency of $209 \mathrm{~cm}^{-1}$. As for the ${ }^{2} \Pi_{r}$ ground state,
TABLE II. Energetic properties (in eV) of $\mathrm{SiCu}, \mathrm{SiCu}^{+}$, and $\mathrm{SiCu}^{-}$.

\begin{tabular}{lccccc}
\hline \hline & $D_{e}(\mathrm{SiCu})$ & $I E_{a}(\mathrm{SiCu})$ & $E A_{a}(\mathrm{SiCu})$ & $D_{e}\left(\mathrm{SiCu}^{+}\right)$ & $D_{e}\left(\mathrm{SiCu}^{-}\right)$ \\
\hline B3LYP $^{\mathrm{a}}$ & 2.043 & 7.246 & 1.475 & 2.836 & 2.193 \\
MP2 $^{\mathrm{a}}$ & 1.783 & 6.874 & 1.325 & 2.165 & 2.054 \\
$\mathrm{QCISD}^{\mathrm{a}}$ & 1.830 & 6.744 & 1.222 & 2.270 & 2.036 \\
$\left.\mathrm{QCISD}^{\mathrm{T}}\right)^{\mathrm{b}}$ & 1.787 & 7.009 & 1.424 & 2.158 & 2.034 \\
\hline \hline
\end{tabular}

${ }^{\mathrm{a} U}$ sing $6-311++\mathrm{G}(d, f)$ basis sets.

${ }^{\mathrm{b}} \mathrm{Using} 6-311++\mathrm{G}(2 d, 2 f)$ basis sets at QCISD/6-311++ $\mathrm{G}(d, f)$ geometry.

we expect that this bond length is too long because $\mathrm{Cu} 3 d$ electron correlation is neglected. However, the excitation energy from the ${ }^{2} \Pi_{r}$ ground state to this state $3.17 \mathrm{eV}$ is close to the $3.22 \mathrm{eV}$ obtained at the QCISD(T) level of theory and to the experimentally observed transition.

As stated earlier, we needed to use the multideterminental based CASSCF $\left(4 a_{1}, 2 b_{1}, 2 b_{1}, 1 a_{1}\right.$ active space within $C_{2 v}$ symmetry) method to treat the ${ }^{2} \Sigma^{+}$and ${ }^{2} \Delta$ states. After so doing and using the CASSCF/MRCISD $(\mathrm{Q})$ energy for the ${ }^{2} \Pi_{r}$ ground state, we find the ${ }^{2} \Delta$ and ${ }^{2} \Sigma^{+}$states (obtained as the lowest roots among the doublet states within $a_{1}$-symmetry) to have excitation energies of 1.21 and 1.76 $\mathrm{eV}$, respectively, above the ground ${ }^{2} \Pi_{r}$ state.

In summery, for $\mathrm{SiCu}$ we found the following state ordering:

$$
\begin{aligned}
& { }^{2} \Pi_{r} \rightarrow{ }^{4} \Sigma^{-} 0.5 \mathrm{eV} \\
& { }^{2} \Pi_{r} \rightarrow{ }^{2} \Delta 1.2 \mathrm{eV} \\
& { }^{2} \Pi_{r} \rightarrow{ }^{2} \Sigma^{+} 1.8 \mathrm{eV} \\
& { }^{2} \Pi_{r} \rightarrow{ }^{2} \Pi_{i} 3.2 \mathrm{eV}
\end{aligned}
$$

\section{B. $\mathrm{SiCu}^{+}$}

We studied three low-lying cation electronic states: ${ }^{1} \Sigma^{+}\left(1 \sigma^{2} 1 \delta^{4} 1 \pi^{4} 2 \sigma^{2} 3 \sigma^{2} 2 \pi^{0}\right), \quad{ }^{3} \Pi_{r}\left(1 \sigma^{2} 1 \delta^{4} 1 \pi^{4} 2 \sigma^{2} 3 \sigma^{1} 2\right.$ $\left.\pi^{1}\right)$, and ${ }^{3} \Sigma^{-}\left(1 \sigma^{2} 1 \delta^{4} 1 \pi^{4} 2 \sigma^{2} 3 \sigma^{0} 2 \pi^{2}\right)$ all of which involve double occupancy for the $3 d$ orbitals of $\mathrm{Cu}$ and of the bonding $2 \sigma$-MO with the two remaining valence electrons distributed throughout the $3 \sigma$ and $2 \pi$ valence orbitals. The ${ }^{1} \Sigma^{+}$state was found to be the ground state at all three (B3LYP, MP2, and QCISD) levels of theory; again all three methods give very similar bond lengths, harmonic frequencies, and dissociation energies.

The calculated adiabatic ionization energy of $\mathrm{SiCu}$ was found to be $7.01 \mathrm{eV}$ at our highest QCISD(T)/6-311++G $(2 d, 2 f)$ level of theory (see Table II). The harmonic frequency $\left(358 \mathrm{~cm}^{-1}\right)$ and dissociation energy $\left(D_{e}=2.16 \mathrm{eV}\right)$ obtained for the ground state of $\mathrm{SiCu}^{+}$, both suggest strong bonding (both are somewhat larger than the corresponding values for the neutral molecule).

The ${ }^{3} \Pi_{r}$ cation state arising from promoting of one electron from the $3 \sigma$-MO into the $2 \pi$-MO was found to be 0.50 $\mathrm{eV}$ above the ground electronic state. $\mathrm{A}^{3} \Sigma^{-}$state was found to be substantially higher in energy, so the ${ }^{1} \Sigma^{+}$state is reasonably well established to be the ground electronic state of $\mathrm{SiCu}^{+}$. 


\section{C. $\mathrm{SiCu}^{-}$}

We studied six low-lying anion electronic states ${ }^{3} \Sigma^{-}(1$ $\left.\sigma^{2} 1 \delta^{4} 1 \pi^{4} 2 \sigma^{2} 3 \sigma^{2} 2 \pi^{2}\right),{ }^{3} \Pi_{i}\left(1 \sigma^{2} 1 \delta^{4} 1 \pi^{4} 2 \sigma^{2} 3 \sigma^{1} 2 \pi^{3}\right),{ }^{1} \Sigma^{+}$ $\left(1 \sigma^{2} 1 \delta^{4} 1 \pi^{4} 2 \sigma^{2} 3 \sigma^{0} 2 \pi^{4}\right), \quad{ }^{1} \Sigma^{+}\left(1 \sigma^{2} 1 \delta^{4} 1 \pi^{4} 2 \sigma^{2} 3 \sigma^{2} 2 \pi^{0} 4\right.$ $\left.\sigma^{2}\right),{ }^{3} \Pi_{r}\left(1 \sigma^{2} 1 \delta^{4} 1 \pi^{4} 2 \sigma^{2} 3 \sigma^{2} 2 \pi^{1} 4 \sigma^{1}\right)$, and ${ }^{5} \Sigma^{-}\left(1 \sigma^{2} 1 \delta^{4} 1\right.$ $\left.\pi^{4} 2 \sigma^{2} 3 \sigma^{1} 2 \pi^{2} 4 \sigma^{1}\right)$ derived using double occupancy for the $3 d$ orbitals of $\mathrm{Cu}$ as well as for the bonding $2 \sigma$-MO with the four other valence electrons distributed throughout the $3 \sigma$, $4 \sigma$, and $2 \pi$ valence orbitals. The ${ }^{1} \Sigma^{+}\left(1 \sigma^{2} 1 \delta^{4} 1 \pi^{4} 2 \sigma^{2} 3 \sigma^{2} 2\right.$ $\pi^{2}$ ) state was not considered here, because we assume that the higher multiplicity ${ }^{3} \Sigma^{-}$state with the same orbital occupancy will be lower in energy.

The ${ }^{3} \Sigma^{-}$state was found to be the ground state at all three (B3LYP, MP2, and QCISD) levels of theory, and again all three methods give very similar bond lengths, harmonic frequencies, and dissociation energies. The calculated adiabatic electron affinity of $\mathrm{SiCu}$ was found to be $1.42 \mathrm{eV}$ at our highest QCISD(T)/6-311++ G(2d,2f ) level of theory (see Table II). Our calculated harmonic frequency $\left(283 \mathrm{~cm}^{-1}\right)$ and dissociation energy into the lowest energy asymptote $\mathrm{Si}^{-}\left({ }^{4} S\right)+\mathrm{Cu}\left({ }^{2} S\right)\left(D_{e}=2.04 \mathrm{eV}\right)$ both suggest strong bonding in the ground state of $\mathrm{SiCu}^{-}$.

${ }^{5} \Sigma^{-}$and ${ }^{3} \Pi_{r}$ states arising from promoting one electron from the $3 \sigma$-MO into the $4 \sigma$-MO and promoting one electron from the $2 \pi$-MO into the $4 \sigma$-MO, respectively, are the lowest excited state with 1.09 and $1.14 \mathrm{eV}$ excitation energies. Three other states ${ }^{1} \Sigma^{+},{ }^{1} \Sigma^{+}$, and ${ }^{3} \Pi_{i}$ are substantially higher in energy, so the ${ }^{3} \Sigma^{-}$state is well established to be the ground electronic state for $\mathrm{SiCu}^{-}$.

\section{SUMMARY}

In summary, we found that the Aufbau principle correctly predicts the ground electronic states for $\mathrm{SiCu}, \mathrm{SiCu}^{+}$, and $\mathrm{SiCu}^{-}$, all of which were found to be relatively strongly bound although the bond lengths, bond energies, and harmonic frequencies vary slightly among these species. These small variations are a result of the nonbonding character of the $2 \pi$-MO, which is almost completely composed of the $\mathrm{Si}$ $3 p$-AO. Of special relevance to the CRLAS study of Scherer et al. ${ }^{1}$ is the fact that $\mathrm{SiCu}$ is likely to have a ${ }^{2} \Pi_{r}$ ground state, not the ${ }^{2} \Sigma$ state suggested earlier. In light of these findings, the analogous CRLAS studied Scherer et al..$^{20,21}$ on the similar $\mathrm{SiAg}$ and $\mathrm{SiAu}$ molecules will also need to be reexamined.

In this work, we used sophisticated ab initio methods to calculate molecular properties of the ground and lowest excited electronic states of $\mathrm{SiCu}, \mathrm{SiCu}^{+}$, and $\mathrm{SiCu}^{-}$. Our results are very similar at the density functional theory (B3LYP), second-order Møller-Plesset perturbation theory (MP2) and quadratic configuration level (QCISD), when valence triple-zeta bases sets augmented by sets of diffuse $s$ and $p$-functions and sets of polarization functions have been used. In all of these calculations, all core electrons were excluded from correlation but the $3 d$-electrons of $\mathrm{Cu}$ were included. Recently we studied the isoelectronic AlZn diatomic molecule at the QCISD/6-311+ $\mathrm{G}^{*}$ and $\operatorname{QCISD}(\mathrm{T}) / 6-311$ $+\mathrm{G}^{*}$ levels of theory and found that triple excitations are very important for proper description of the vibrational frequency. ${ }^{22}$ We therefore reexamined the geometries and frequencies of $\mathrm{SiCu}, \mathrm{SiCu}^{+}$, and $\mathrm{SiCu}^{-}$at $\mathrm{QCISD}(\mathrm{T}) / 6-311$ $+\mathrm{G}^{*}$ level of theory. The calculated molecular parameters at this level of theory: $r_{e}=2.235 \AA$ and $\omega_{e}=325 \mathrm{~cm}^{-1}$ for $\mathrm{SiCu}$ $\left({ }^{2} \Pi_{r}\right) ; r_{e}=2.258 \AA$ and $\omega_{e}=311 \mathrm{~cm}^{-1}$ for $\mathrm{SiCu}^{+}\left({ }^{1} \Sigma^{+}\right)$and $r_{e}=2.268 \AA$ and $\omega_{e}=270 \mathrm{~cm}^{-1}$ for $\mathrm{SiCu}^{-}\left({ }^{3} \Sigma^{-}\right)$are very close to those found at the QCISD/6-311+G* level of theory. Therefore for the species studied here triple excitations were found not to be important.

Finally, we would like to stress that while diatomic molecules are the simplest chemical species, most have not yet been fully characterized either theoretically or experimentally. Recently two of us (A.I.B. and J.S.) created three diatomic periodic tables (main group dimers, transition metal dimers, and mixed dimers), ${ }^{23}$ which summarize all that is known about the term symbols, bond lengths, and dissociation energies of diatomic species. In these tables there are many species for which nothing or little is known. Taking into account the importance of high quality data on diatomic molecules for understanding chemical bonding among atoms in larger molecules, we hope that more efforts will be made to fill in the missing data in these Tables.

\section{ACKNOWLEDGMENTS}

The authors wish to thank Professor Michael Morse and Professor Richard Barrow for bringing to their attention the possibility that $\mathrm{SiCu}$ might have a ${ }^{2} \Pi$ rather than ${ }^{2} \Sigma$ ground electronic state. This theoretical work was supported by NSF Grant No. CHE9116286. The Saykally CRLAS studies were supported by the AFORS Grant No. F49620-96-1-0411.

${ }^{1}$ J. J. Scherer, J. B. Paul, C. P. Collier, and R. J. Saykally, J. Chem. Phys. 102, 5190 (1995).

${ }^{2}$ A. D. McLean and G. S. Chandler, J. Chem. Phys. 72, 5639 (1980).

${ }^{3}$ R. Krishnan, J. S. Binkley, R. Seeger, and J. A. Pople, J. Chem. Phys. 72, 650 (1980).

${ }^{4}$ A. J. H. Wachters, J. Chem. Phys. 52, 1033 (1970).

${ }^{5}$ P. J. Hay, J. Chem. Phys. 66, 4377 (1977).

${ }^{6}$ K. Raghavachari and G. W. Trucks, J. Chem. Phys. 91, 1062 (1989).

${ }^{7}$ T. H. Dunning, Jr. and P. J. Hay, in Modern Theoretical Chemistry, edited by H. F. Schaefer III (Plenum, New York, 1976), pp. 1-28.

${ }^{8}$ C. Lee, W. Yang, and R. G. Parr, Phys. Rev. B 37, 785 (1988).

${ }^{9}$ A. D. Becke, J. Chem. Phys. 98, 5648 (1993).

${ }^{10}$ J. A. Pople, R. Seeger, and R. Krishnan, Int. J. Quant. Chem. Symp. 11, 149 (1977).

${ }^{11}$ J. A. Pople, M. Head-Gordon, and K. Raghavachari, J. Chem. Phys. 87, 5968 (1987).

${ }^{12}$ H. B. Schlegel, J. Chem. Phys. 84, 4530 (1984).

${ }^{13}$ Gaussian 94 (Revision A1), M. J. Frisch, G. M. Trucks, H. B. Schlegel, P. M. W. Gill, B. G. Johnson, M. A. Robb, J. R. Cheeseman, T. A. Keith, G. A. Petersson, J. A. Montgomery, K. Raghavachari, M. A. Al-Laham, V. G. Zakrzewski, J. V. Ortiz, J. B. Foresman, J. Cioslowski, B. B. Stefanov, A. Nanayakkara, M. Challacombe, C. Y. Peng, P. Y. Ayala, W. Chen, M. W. Wong, J. L. Andres, E. S. Replogle, R. Gomperts, R. L. Martin, D. J. Fox, J. S. Binkley, D. J. Defrees, J. Baker, J. J. P. Stewart, M. HeadGordon, C. Gonzales, and J. A. Pople, Gaussian, Inc., Pittsburgh, PA, 1995.

${ }^{14}$ (a) K. Andersson, P.-A. Malmqvist, B. O. Roos, A. J. Sadlej, and K. Wolonski, J. Phys. Chem. 94, 5483 (1990); (b) K. Andersson, P.-A. Malmqvist, and B. O. Roos, J. Chem. Phys. 96, 1218 (1992).

${ }^{15}$ (a) B. O. Roos, Chem. Phys. Lett. 15, 153 (1972); (b) I. Shavitt, Int. J. Quantum Chem. Symp. 11, 131 (1977), 12, 5 (1978); (c) P. E. M. Siegbahn, J. Chem. Phys. 72, 1647 (1980).

${ }^{16}$ K. Pierloot, B. Dumez, P.-O. Widmark, and B. O. Roos, Theor. Chim. Acta 90, 87 (1995).

${ }^{17}$ MOLCAS Version 3, K. Anderson, M. R. A. Blombeg, M. P. Fulscher, V. 
Kello, R. Lindh, P.-A. Malmqvist, J. Noga, J. Olsen, B. O. Roos, A. J. Sadlej, P. E. M. Siegbahn, M. Urban, and P.-O. Widmark, University of Lund, Sweden, 1994.

${ }^{18}$ E. R. Davidson, in The World of Quantum Chemistry, edited by R. Daudel (Reidel, Dortrecht, 1974), p. 17.

${ }^{19}$ S. R. Langhoff and E. R. Davidson, Int. J. Quantum Chem. 8, 61 (1974).

${ }^{20}$ J. J. Scherer, J. B. Paul, C. P. Collier, and R. J. Saykally, J. Chem. Phys. 103, 113 (1995).
${ }^{21}$ J. J. Scherer, J. B. Paul, C. P. Collier, and R. J. Saykally, J. Chem. Phys. 103, 9187 (1995).

${ }^{22}$ A. I. Boldyrev and J. Simons, Mol. Phys. 92, 365 (1997).

${ }^{23}$ A. I. Boldyrev and J. Simons, Periodic Table of Diatomic Molecules. Part A: Diatomics of Main Group Elements. Part B: Diatomics Composed of One Main Group Element and One Transion Metal Atom. Part C: Diatomics of Transition Metals (Wiley, London, 1997). 